\title{
Application of Monte Carlo techniques for propagation of cross section uncertainties to actinide inventory in ADS transmuters: comparison with sensitivity analysis
}

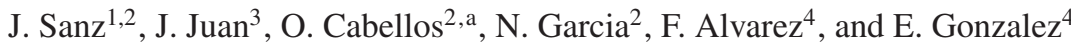 \\ 1 Department of Power Engineering, UNED, Madrid, Spain \\ 2 Institute of Nuclear Fusion, UPM, Madrid, Spain \\ 3 Department of Statistics, UPM, Madrid, Spain \\ ${ }^{4}$ CIEMAT, Madrid, Spain
}

\begin{abstract}
A comprehensive study is performed in order to evaluate the impact of activation cross section uncertainties on the actinide composition of the irradiated fuel in representative ADS irradiation scenarios. Some of the most recent sources/compilations of uncertainty data are used, and the results obtained from them compared. The ANL covariance matrices are taken as reference data for the calculations. The complete set of cross section uncertainties provided in the EAF2005 data library are also used for comparison purposes. In this study, the inventory code $\mathrm{ACAB}$ is used to analyze the following questions: impact of different correlation structures using fixed uncertainties/variances; effect of the irradiation time/burn-up on the concentration uncertainties; and applicability of Monte Carlo (MC) and sensitivity-uncertainty (SU) approaches for all the range of burn-up/irradiation times of interest in ADS designs.
\end{abstract}

\section{Introduction}

The concept of a subcritical system driven by an external source of neutrons provided by an accelerator ADS (Accelerator Driven System) has become the object of EU Integrated Project EUROTRANS. This paper is within the frame of the NUDATRA (Nuclear Data for Transmutation) Domain of the Project. One of the objectives in NUDATRA is to evaluate the impact of nuclear data uncertainties on relevant fuel cycle and repository parameters. Here, as one of the parts of the problem, we estimate the propagation of activation cross section uncertainties to the actinide concentrations of the irradiated fuel.

The most comprehensive ADS study [1] addressing this kind of problems was performed using sensitivity-uncertainty (SU) analysis. The results were obtained for 1-year irradiation period, using cross section data uncertainties produced by ANL. More recently, nuclear data uncertainty impacts were assessed for other future nuclear systems [2], using as uncertainty data the ANL covariance matrix [3] obtained by updating the uncertainty data used in [1]. In our recent ADS study [4], a broad range of burn-up levels are explored. The Monte Carlo approach is used for error propagation, and the results are compared with those obtained with the SU method, in order to see if non-linear effects are relevant for the ADS high burnup levels. The ANL covariance matrix is used as reference, but also other correlation structures are considered. Uncertainty data from the EAF2005 [5] library are also used for comparison purposes. Here the main results of the study are presented.

\footnotetext{
${ }^{a}$ Presenting author, e-mail: cabellos@din.upm.es
}

\section{System used and calculational tools}

The system used in the analysis has been defined by the CIEMAT group [6], and it is one of the two ADS transmuters considered in the RED-IMPACT project [7]. The basic ADS characteristics are: fuel (TRU-Zr) N, core cooled by lead-bismuth eutectic in forced convection, total fuel burn-up $150 \mathrm{GWd} / \mathrm{THM}$, thermal power $850 \mathrm{MW}$, and initial total mass of actinides 3.64 tonnes. The initial isotopic composition for actinides is shown in column 2 of table 1 (for a mass of $16.6 \mathrm{~kg}$, corresponding to the calculation cell used in MCNP).

The problem of reference for us is to estimate uncertainties in the actinide inventory at the end of a 500-day irradiation period. In the calculations, we have used for all the irradiation period both a constant neutron spectrum (average energy $\langle\mathrm{E}\rangle=0.365 \mathrm{MeV})$ and flux intensity $\left(5.73 \times 10^{15} \mathrm{n} / \mathrm{cm}^{2} \cdot \mathrm{s}\right)$. With this irradiation conditions we reach a burn-up of $\approx 175$ $\mathrm{GWd} / \mathrm{t}$ at 500 days. The assumed neutron flux and spectrum are taken from detailed neutronics calculations in the ADS CIEMAT design, corresponding to a given cell in the interior of the core and at 250 days of irradiation.

The burnup level effect in uncertainty estimates of concentrations is investigated considering irradiation times ranging from 250 to 1000 days. The constant neutron environment given above is assumed during irradiation.

The ANL covariance matrix is used as reference uncertainty data. This matrix is probably at present the data set best suited for uncertainty estimates in actinide inventory prediction. It should be understood as consisting of the set of uncertainties (diagonal values) and the set of correlations. Only energy correlation is considered, and the same partial energy (PE) correlation matrix is assumed for all isotopes and reactions. 
As correlations data are at present very poorly known, we have generated several parametric correlation matrices associated to a simple correlation model. The rational followed in the generation of these matrices is as follows. Let us assume that the energy range are divided in $G$ groups and let $\left[E_{1}, E_{2}, \ldots, E_{G}\right]$ be the energy mean values or centre points of each group. The correlation among the groups with energies $E_{i}$ and $E_{j}$ is defined by the equation

$$
c_{i j}=\exp \left[-\theta\left|\log E_{i}-\log E_{j}\right|\right]
$$

where $\theta$ is a positive parameter between 0 and $\infty$, that can be considered as a energy-correlation range parameter. When $\theta$ is large, the correlations are low and vice versa, when $\theta$ is small, the correlations are high. In the extreme cases, $\theta=\infty$, all the coefficients $c_{i j}$ are zero (no correlation) and when $\theta=0$, all the coefficients are 1 (full correlation). For a given value of $\theta$, the correlation $c_{i j}$ decreases as the groups are more distant. For the fixed ANL variance/diagonal data set, the effect of the covariance structure in the actinide inventory will be explored using in addition to the ANL PE correlation matrix, a series of these correlation parametric matrices. These matrices are produced in the ANL 15-group structure.

The inventory code ACAB has been used for isotopic inventory predictions. This code uses two different approaches to estimate the propagation of cross section uncertainties to the isotopic inventory of the irradiated fuel and associated fuel cycle parameters: sensitivity-uncertainty (SU) and Monte Carlo (MC) uncertainty analyses. It is able to deal with multigroup covariance matrices in any arbitrary group structure and with any kind of correlations. The MC approach, one of the singular features of code, is based on using a log normal joint probability distribution of the cross sections for each reaction. Here the results are obtained for a 1000 history-sampling.

The activation cross section expectation/best-estimated values have been taking from the EAF-2005 cross section library, and processed to the required ANL 15-multigroup structure. Cross section uncertainties from EAF-2005 have been also used in the study (see section 3.3).

We have selected the 24 actinides given in the first column of table 1 as the most of concern in regards to transmutation potential and fuel cycle integral parameters. The nominal values (no uncertainties considered) of the actinide concentrations at the end of the 500-day irradiation period are given in the third column of table 1.

According to the actinides included in the ANL uncertainty data set (ranging (from Th-232 to Cm-245), uncertainty concentrations obtained with ANL data (table 1 in columns 4-11; and table 2) are meaningful for the first 19 actinides of the two tables (from U-234 to Cm-245) and can be also of some meaning for $\mathrm{Cm}-246$, since it is mainly generated from Cm-245. For the other 4 nuclides $(\mathrm{Cm} \mathrm{247,} \mathrm{Cm} 248$, Bk-249, and Cf-50) the results are meaningless. On the other hand, estimates of concentration uncertainties (column 12 of table 1) obtained using the EAF-2005 cross section uncertainty data are meaningful for all the nuclides, since this library includes uncertainty information for all the actinides of interest. It is worth noting that regarding target accuracy on actinide concentration estimates (mainly related with the
ADS transmutation potential), the assigned value is taken to be $\pm 5 \%[1,2,4]$.

\section{Uncertainty evaluation}

\subsection{Results from ANL covariance matrices and parametric correlation matrices}

For the reference 500-day irradiation problem, different set of results have been obtained using the MC approach, and are provided in table 1 . In the first set, only the "diagonal" values of the ANL covariance matrices are used (any kind of correlations is neglected). The results obtained are given in column 4 of table 1 . In the second set, the full ANL covariance matrices are considered, i.e., including the energy correlation assigned in [3]. The results are provided in column 11. The third set uses a series of correlation parametric matrices, generated according with equation (1) for the following values of $\theta: 1,0.5,0.25,0.1,0.05$ and 0 . The results are provided in columns 5-10.

Results given in columns 4-11 show that: i) most of the nuclides exhibit concentration uncertainties below the target value of $5 \%$; ii) there are a few nuclides with uncertainty values a little higher than $5 \%$ (in this group the nuclide with a higher uncertainty in the concentration is Am-242m.; and iii) there are two nuclides, Cm-243 and Cm-245, that clearly show a concentration uncertainty far above $5 \%$.

It is seen that the nature of the introduced correlations is very relevant to estimate the overall uncertainty in the concentration of all the nuclides in general. The need of more scientifically based correlation data is clearly justified. It is also seen that the uncertainties in concentrations obtained assuming the ANL partial energy correlation matrix are very similar to those obtained assuming a correlation range parameter $\theta$ around to 0.5 (only a little bit higher than 0.5 ).

\subsection{Irradiation time effect on concentration uncertainties and applicability of MC and SU approaches}

In this section, we compute the concentration uncertainties at the end of different irradiation times of relevance for ADS designs, with two purposes: i) examine the irradiation time effect on the concentration uncertainties, and ii) explore the applicability of MC and SU approaches within the range of these different burn-up/irradiation time situations.

Regarding the first question, the Monte Carlo approach is used to obtain uncertainties in the concentrations at the end of the following irradiation times: 250, 500, 750 and 1000 days. The cross section uncertainty data (diagonal values of covariance matrix) from the ANL matrix are used. And different kinds of correlations are considered: uncorrelated, the ANL PE correlation and the abovementioned parametric correlations $(\theta=1, \theta=0.5, \theta=0.25, \theta=0.1, \theta=0.05$ and $\theta=0)$.

In table 2, columns 3, 5, 7 and 9, we summarize the results for the ANL partial energy correlation case. These results show that the impact of the irradiation time on the 
Table 1. Uncertainty evaluation using ANL uncertainties (diagonal values, column 4) and different correlation matrices (ANL PE, column 11, and parametric correlations, columns 5-10). The relative error (ratio between standard deviation and the mean, in \%) are calculated by the MC approach. Results using EAF2005 uncertainties are also given (column 12). Results are referred at the end of 500-day irradiation.

\begin{tabular}{|c|c|c|c|c|c|c|c|c|c|c|c|}
\hline \multirow[b]{3}{*}{ Isotope } & \multirow{3}{*}{$\begin{array}{c}\text { Initial } \\
\left(10^{23} \text { at. }\right)\end{array}$} & \multirow{3}{*}{$\begin{array}{l}\text { Final } \\
\left(10^{23} \text { at. }\right)\end{array}$} & \multirow{3}{*}{$\begin{array}{c}\text { No } \\
\text { correl. }\end{array}$} & \multicolumn{8}{|c|}{ Relative error of the final concentrations (\%) } \\
\hline & & & & \multicolumn{6}{|c|}{ Theta $(\theta)$} & \multirow{2}{*}{$\begin{array}{l}\text { ANL } \\
\text { correl. }\end{array}$} & \multirow{2}{*}{$\begin{array}{l}\text { EAF } \\
2005\end{array}$} \\
\hline & & & & 1 & 0.5 & 0.25 & 0.1 & 0.05 & 0 & & \\
\hline${ }^{234} \mathrm{U}$ & 4.30 & 3.74 & 1.13 & 1.52 & 1.73 & 2.01 & 2.19 & 2.36 & 2.50 & 1.69 & 4.47 \\
\hline${ }^{235} \mathrm{U}$ & 1.08 & 0.99 & 2.30 & 3.11 & 3.72 & 4.71 & 5.19 & 5.69 & 5.93 & 3.52 & 13.05 \\
\hline${ }^{236} \mathrm{U}$ & 1.50 & 1.45 & 0.90 & 1.24 & 1.55 & 1.79 & 2.04 & 2.22 & 2.27 & 1.47 & 1.94 \\
\hline${ }^{238} \mathrm{U}$ & 0.01 & 0.009 & 0.49 & 0.67 & 0.83 & 0.96 & 1.15 & 1.23 & 1.26 & 0.81 & 1.69 \\
\hline${ }^{237} \mathrm{~Np}$ & 17.95 & 10.84 & 1.87 & 2.53 & 3.00 & 3.54 & 4.18 & 4.18 & 4.51 & 3.01 & 7.10 \\
\hline${ }^{238} \mathrm{Pu}$ & 32.10 & 28.73 & 1.96 & 2.63 & 3.13 & 3.86 & 4.44 & 4.57 & 5.10 & 2.93 & 4.89 \\
\hline${ }^{239} \mathrm{Pu}$ & 46.77 & 29.41 & 1.30 & 1.73 & 2.09 & 2.51 & 2.88 & 2.99 & 3.20 & 1.98 & 5.47 \\
\hline${ }^{240} \mathrm{Pu}$ & 137.40 & 113.53 & 0.81 & 1.09 & 1.40 & 1.69 & 1.92 & 2.04 & 2.21 & 1.34 & 2.31 \\
\hline${ }^{241} \mathrm{Pu}$ & 28.12 & 24.21 & 3.07 & 4.10 & 5.14 & 6.16 & 7.26 & 7.87 & 8.36 & 4.85 & 9.90 \\
\hline${ }^{242} \mathrm{Pu}$ & 61.44 & 55.35 & 0.81 & 1.04 & 1.25 & 1.49 & 1.79 & 1.89 & 1.94 & 1.24 & 2.44 \\
\hline${ }^{243} \mathrm{Pu}$ & & 0.0005 & 3.80 & 4.89 & 6.20 & 7.34 & 8.96 & 9.75 & 9.56 & 5.83 & 12.18 \\
\hline${ }^{244} \mathrm{Pu}$ & & 0.0004 & 3.48 & 4.25 & 5.74 & 6.49 & 7.91 & 8.16 & 8.66 & 5.22 & 14.09 \\
\hline${ }^{242} \mathrm{Am}$ & 26.55 & 16.09 & 1.89 & 2.55 & 3.16 & 3.60 & 4.15 & 4.31 & 4.86 & 2.91 & 7.52 \\
\hline${ }^{242 \mathrm{~m}} \mathrm{Am}$ & 1.49 & 0.98 & 4.27 & 5.74 & 7.49 & 8.58 & 9.49 & 10.07 & 10.85 & 6.04 & 14.72 \\
\hline${ }^{243} \mathrm{Am}$ & 24.39 & 21.37 & 1.98 & 2.50 & 3.26 & 3.66 & 4.43 & 4.74 & 4.96 & 3.00 & 7.46 \\
\hline${ }^{242} \mathrm{Cm}$ & 0.02 & 2.87 & 3.20 & 4.26 & 5.22 & 5.86 & 6.81 & 7.12 & 7.68 & 4.87 & 11.17 \\
\hline${ }^{243} \mathrm{Cm}$ & 0.22 & 0.32 & 14.78 & 19.00 & 24.21 & 27.13 & 32.09 & 35.24 & 35.95 & 22.91 & 28.30 \\
\hline${ }^{244} \mathrm{Cm}$ & 20.59 & 23.12 & 3.72 & 4.48 & 5.54 & 6.65 & 7.49 & 7.73 & 8.22 & 5.43 & 6.89 \\
\hline${ }^{245} \mathrm{Cm}$ & 5.92 & 5.52 & 11.62 & 14.17 & 18.35 & 21.59 & 25.51 & 26.18 & 28.45 & 16.68 & 15.06 \\
\hline${ }^{246} \mathrm{Cm}$ & 3.82 & 3.81 & 3.38 & 4.36 & 5.73 & 7.05 & 7.53 & 8.38 & 8.90 & 5.40 & 7.71 \\
\hline${ }^{247} \mathrm{Cm}$ & 1.05 & 0.90 & 0.88 & 1.15 & 1.50 & 1.84 & 1.97 & 2.20 & 2.33 & 1.43 & 15.58 \\
\hline${ }^{248} \mathrm{Cm}$ & & 0.14 & 0.31 & 0.41 & 0.53 & 0.64 & 0.69 & 0.77 & 0.82 & 0.50 & 31.27 \\
\hline${ }^{249} \mathrm{Bk}$ & & 0.0004 & 0.18 & 0.24 & 0.31 & 0.38 & 0.41 & 0.46 & 0.49 & 0.30 & 39.78 \\
\hline${ }^{250} \mathrm{Cf}$ & & 0.00005 & 0.11 & 0.14 & 0.18 & 0.22 & 0.24 & 0.26 & 0.28 & 0.17 & 50.33 \\
\hline
\end{tabular}

uncertainties is very important. It is also found that the uncertainties in the concentrations increase with the irradiation time for nearly all the nuclides. Yet there are three exceptions: $\mathrm{Pu}-243, \mathrm{Pu}-244$ and $\mathrm{Cm}-242$ (which is the one exhibiting the highest decreasing in the uncertainty from 250 to 1000 days of irradiation). The results obtained using the other correlation matrices show that the correlation structure effect is very important in the prediction of the concentrations at the end of all the irradiation times considered [4].

As for the second question raised in this section, the abovementioned set of problems dealt with the MC approach, have been also addressed by means of the sensitivity-uncertainty analysis method. We summarize in table 2, columns 2, 4, 6 and 8 , the results obtained by SU analysis for the ANL partial energy correlation case. From table 2, when comparing the results obtained with SU and MC methods, it can be concluded that both approaches are acceptable to deal with the range of irradiation times of interest for ADS applications. The same conclusion is reached when using any of the correlation matrices considered [4].

\subsection{Comparison of uncertainty evaluations using EAF2005/UN and ANL data}

When comparing results of calculations using ANL vs. EAF2005/UN uncertainty data (see columns 11 and 12), we found significant differences in the concentration uncertainties at the end of ADS representative burnup levels.

As for ANL results, there are only two nuclides that clearly show a concentration uncertainty far above the required target accuracy of 5\%, while using EAF2005 uncertainty data, there area a relatively large number of actinides (more than $50 \%$ of the actinides considered) with a concentration uncertainty higher than $5 \%$. The differences in ${ }^{247} \mathrm{Cm},{ }^{248} \mathrm{Cm},{ }^{249} \mathrm{Bk}$ and ${ }^{250} \mathrm{Cf}$ results can be explained by the lack of uncertainty information for these nuclides in the ANL uncertainty set. The differences for the other nuclides $\left({ }^{235} \mathrm{U},{ }^{243} \mathrm{Pu},{ }^{244} \mathrm{Pu},{ }^{242 \mathrm{~m}} \mathrm{Am}\right.$, etc.) are due to the larger uncertainties associated to the EAF2005/UN file [4].

\section{Conclusions and ongoing work}

The impact of potential activation cross section uncertainties on the actinide inventory is assessed for a broad range of burn-up/irradiation times of interest in ADS designs. Some of the uncertainty data sets most recently produced are used in the evaluation, and the results obtained from them compared. We conclude that more effort in defining diagonal/uncertainty data for actinide prediction is needed. In addition, the need of much more scientifically based correlation data is clearly justified. When comparing results of calculations using ANL vs. EAF2005/UN uncertainty data, we found very significant differences in the concentration uncertainties. 
Table 2. Relative errors (in \%) for actinide concentrations at the end of the following irradiation times: 250, 500, 750 and 1000 days. Comparison between sensitivity/uncertainty and Monte Carlo methods. ANL PE correlation is used.

\begin{tabular}{|c|c|c|c|c|c|c|c|c|}
\hline \multirow[b]{2}{*}{ Isotope } & \multicolumn{2}{|c|}{$250 \mathrm{~d}$} & \multicolumn{2}{|c|}{$500 \mathrm{~d}$} & \multicolumn{2}{|c|}{$750 \mathrm{~d}$} & \multicolumn{2}{|c|}{$1000 \mathrm{~d}$} \\
\hline & $\overline{\mathrm{SU}}$ & M.C. & SU & M.C. & SUr & M.C. & SUr & M.C. \\
\hline${ }^{234} \mathrm{U}$ & 0.83 & 0.86 & 1.64 & 1.69 & 2.41 & 2.51 & 3.16 & 3.22 \\
\hline${ }^{235} \mathrm{U}$ & 2.05 & 2.07 & 3.44 & 3.52 & 4.36 & 4.48 & 4.97 & 5.08 \\
\hline${ }^{236} \mathrm{U}$ & 0.75 & 0.79 & 1.47 & 1.47 & 2.15 & 2.15 & 2.80 & 2.79 \\
\hline${ }^{238} \mathrm{U}$ & 0.42 & 0.45 & 0.80 & 0.81 & 1.14 & 1.15 & 1.44 & 1.45 \\
\hline${ }^{237} \mathrm{~Np}$ & 1.46 & 1.48 & 2.91 & 3.01 & 4.34 & 4.33 & 5.74 & 5.53 \\
\hline${ }^{238} \mathrm{Pu}$ & 1.63 & 1.69 & 2.93 & 2.93 & 4.00 & 4.17 & 4.95 & 5.09 \\
\hline${ }^{239} \mathrm{Pu}$ & 0.97 & 0.95 & 1.91 & 1.98 & 2.81 & 2.87 & 3.65 & 3.70 \\
\hline${ }^{240} \mathrm{Pu}$ & 0.65 & 0.69 & 1.27 & 1.34 & 1.88 & 1.95 & 2.48 & 2.52 \\
\hline${ }^{241} \mathrm{Pu}$ & 2.96 & 3.04 & 4.84 & 4.85 & 6.00 & 5.94 & 6.68 & 6.58 \\
\hline${ }^{242} \mathrm{Pu}$ & 0.67 & 0.61 & 1.30 & 1.24 & 1.90 & 1.75 & 2.47 & 2.33 \\
\hline${ }^{243} \mathrm{Pu}$ & 6.33 & 6.19 & 6.08 & 5.83 & 5.95 & 5.46 & 5.95 & 5.19 \\
\hline${ }^{244} \mathrm{Pu}$ & 5.83 & 5.71 & 5.34 & 5.22 & 4.95 & 4.88 & 4.64 & 4.50 \\
\hline${ }^{242} \mathrm{Am}$ & 1.49 & 1.52 & 2.91 & 2.91 & 4.25 & 4.24 & 5.50 & 5.72 \\
\hline${ }^{242 \mathrm{~m}} \mathrm{Am}$ & 3.62 & 3.47 & 6.15 & 6.04 & 7.99 & 8.12 & 9.40 & 9.19 \\
\hline${ }^{243} \mathrm{Am}$ & 1.64 & 1.62 & 2.98 & 3.00 & 4.05 & 4.08 & 4.90 & 4.94 \\
\hline${ }^{242} \mathrm{Cm}$ & 5.34 & 5.45 & 4.69 & 4.87 & 4.05 & 4.23 & 3.54 & 3.71 \\
\hline${ }^{243} \mathrm{Cm}$ & 13.54 & 15.16 & 21.48 & 22.91 & 25.03 & 25.69 & 27.31 & 27.69 \\
\hline${ }^{244} \mathrm{Cm}$ & 2.85 & 3.03 & 5.05 & 5.43 & 6.86 & 7.36 & 8.43 & 9.10 \\
\hline${ }^{245} \mathrm{Cm}$ & 9.77 & 10.58 & 16.16 & 16.68 & 20.02 & 19.95 & 22.22 & 23.55 \\
\hline${ }^{246} \mathrm{Cm}$ & 2.54 & 2.85 & 4.86 & 5.40 & 7.15 & 7.77 & 9.38 & 10.06 \\
\hline${ }^{247} \mathrm{Cm}$ & 0.35 & 0.39 & 1.26 & 1.43 & 2.60 & 2.88 & 4.25 & 4.63 \\
\hline${ }^{248} \mathrm{Cm}$ & 0.12 & 0.13 & 0.44 & 0.50 & 0.93 & 1.04 & 1.57 & 1.72 \\
\hline${ }^{249} \mathrm{Bk}$ & 0.06 & 0.07 & 0.26 & 0.30 & 0.59 & 0.67 & 1.06 & 1.16 \\
\hline${ }^{250} \mathrm{Cf}$ & 0.04 & 0.04 & 0.15 & 0.17 & 0.34 & 0.38 & 0.59 & 0.66 \\
\hline
\end{tabular}

The applicability of both MC and SU approaches is found acceptable to deal with all the range of irradiation times. However, we recommend using the Monte Carlo technique when computing the overall/global effect of a complete set of uncertainties on the isotopic concentrations. The MC method allows us to deal with all the situations without any simplification and with affordable computing time.

The ongoing work is aimed to: i) evaluate uncertainties in the other nuclear fuel cycle related parameters (decay heat, neutron source, and dose); ii) carry out the analysis of the uncertainty results for all the parameters (isotopic concentrations and fuel cycle related parameters); and finally, iii) provide if needed, the table of required accuracies for cross section data.

This work is supported by the EU EUROTRANS Project, and by the Spanish Organism CIEMAT (Centro de Investigaciones Energéticas, Medioambientales y Tecnológicas).

\section{References}

1. G. Aliberti, G. Palmiotti, M. Salvatores, C.G. Stenberg, Nucl. Sci. Eng. 146, 13 (2004).

2. G. Aliberti, G. Palmiotti, M. Salvatores, T.K. Kim, T.A. Taiwo, M. Anitescu, I. Kodeli, E. Sartori, J.C. Bosq, J. Tommasi, Ann. Nucl. Energy 33, 700 (2006).

3. G. Palmiotti, M. Salvatores, Nuclear Energy Agency Report, JEFDOC 1063 Rev.1 January, 2005.

4. J. Sanz et al., Deliverable D5.10, Domain DM5 NUDATRA, EUROTRANS Project, February 2007.

5. R.A. Forrest, J. Kopecky, J.-Ch. Sublet, EURATOM/ UKAEA Fusion Association, UKAEA FUS 515 (2005).

6. E. González, M. Embid-Segura, A. Pérez-Parra, Centro Investigaciones Energéticas, Medioambientales y Tecnológicas DFN/TR01/PC-01, CIEMAT (2001); and Global'01, September 9-13, Paris, France, 2001.

7. L. Boucher et al., FP6 RED-IMPACT Deliverable 1.4. Contract No. FI6W-CT-2004-002408, Draft version, November 2004.

8. J. Sanz, Lawrence Livermore National Laboratory Report, UCRLMA-143238, February 2000. 